\section{THE TREATMENT OF WOUNDS BY FLAVINE. BY}

E. I. PILCHER, F.R.C.S., D.S.O., Colonel A.M.S., AND

A. J. HULL, F.R.C.S., Lieur.-Colonel R.A.M.C.

We have seen lately in the Lancet and British Medical Journat two articles impugning the value of flaviue and its congeners as antiseptics; and as we have used it largely in military practice and are confirmed in our good opinion of it as time passes, we think it would be something of the nature of an act of ingratitude if we allowed an excellent wound dressing to fall into discredit for want of support from its friends.

'The first of the articles referred to takes flavine from the laboratory point of view and criticizes the technique by which its efficacy as against bacteria in vitro was established. With these points we may leave Dr. Browning and his collaborators to deal. But the second article which appeared in the British Medical Journal of December 29th, 1917, and which takes us to the bedside of some fifty selected cases, we feel more competent to criticize. Its lucid exposition and fair and candid treat. ment of the observed facts make it easy to do so.

Two main theses are proposed in this article. First, a comparison is made between flavine and Carrel-Dakin treatment, in which the former, though not inferior at all points, is yet made, on the criteria chosen, to take a second place; and secondly, by histological examination flavine is shown to delay the process of repair, to allow organisms to remain on the wound surface, and to prevent epithelial ingrowth.

Now we hold that a comparison of any method with that of Messrs. Carrel and Dakin is not quite fair until the following facts are remembered: When these workers first showed that infected wounds could be cleansed by continuous washing with hypochlorite solution, they also pointed out the great difficulties of the method, the meticulous care required in the preparation of the reagent, in the protection of the surrounding skin, in the surgical ritual of the dressing, and so on. More than this, they were the first to set the ideal of ultimate wound suture, for we are sure no English surgeon had ever thought it possible before. Both these points-the difficulty of technique and the high ideal of repair-are characteristic of the Carrel-Dakin method, and explain why, though it is one of the most brilliantly successful ways of treating infected wounds in war, it is also one of the most difficult to carry out to full success. It is only in certain hands that it is really successful, and when it fails it fails badly.

One of us, who has opportunities of constantly visiting ten large hospitals, is prepared to affirm that not 10 per cent. of the surgeons whose work he sees have the requisite time in periods of stress, even though they possess the requisite patience of technique, to achieve anything like, for example, the success of the series of fifty cases quoted in this article. In making the com. parison between the two series, the authors have heavily weighted the scales against the Carrel-Dakin method. This series is made up of " much more seriously wounded men," including cases considered too severe for experimental treatment with flavine, and the wounds submitted to the flavine treatment had previously for the most part been treated by the Carrel-Dakin method, and yet nearly double the percentage of suture cases is shown, the number of cases evacuated liealed is two and a half times as many, and so on. This is plainly a series which has been in the hands of a master.

But to any observer who has opportunities of seeing the Carrel-Dakin method as performed by many hands, the above results seem a triumpl of method indeed, but above all a triumph of individual technique. Results which we assert can only be obtained by an expert with much time and comparatively few cases are contrasted with a method which, to take a phrase from mechanics, is practically fool proof, for we suppose that, allowing adequate previous surgical treatment, any dresser can fill a wound cavity with gauze dipped in 1 in 1,000 solution of flavine.

We can only say that in the many hundreds of cases (rather more than 5,000 at present) so treated in the hospitals under the command of one of us, we have found that for ease of preparation and application, rapidity when dealing with large numbers of cases, complete absence of surgical fidget, early cleaning of the wounds, and abatement of coustitutional reaction to absorption, flavine (and its congener brilliant green) is an admirable application under all circumstances, but especially where surgeons are few, time is short, and wounds are many. But, even when contrasted with the Carrel-Dakin series, the series of fifty flavine cases does not come badly out of the test. We note that temperature falls early; that more than onethird reach the difficult suture ideal; and that, though there is delay in the process of healing, there is some diminution in the local and general reaction to infection.

We remember, of course, that the flavine series was in the same capable surgical liands as the other scries, though this consideration must be discounted by the greater ease of application of flavine dressings. To sum up our criticism: we hold that, putting aside any question of the permanence and effectiveness of the antiseptic solution used in the Carrel-Dakin treatment (though this has been called in question), the method itself is not of universal application, entirely on account of its inlierent difficulties of technique. If this is allowed, any simpler method which can be effectively used by less skilled workers, and which approaches in efficiency, as judged by the suture standard, the Carrel-Dakin method, is worthy in spite of minov drawbacks, of an extended trial in the army. We claim to have already passed through such a trial, and have made up our minds on the point. It only remains to add that in the 5,000 or more wounds under our observation no slin irritation has been observed, and we suggest that it is possible that the irritation observed in the cases quoted was due to the previous treatment by the hypochlorite method.

Coming next to the histological part of the article quoted, the appearances observed in the excellently reproduced series of sections given is conclusive evidence of a certain amount of surface destruction in wounäs treated by flavine. This is clinically apparent in a few cases only in our experience. In these cases a fibrinous pellicle forms in the wound, and we have always been used to attribute this appearance to the use of too strong solution. There are no doubt individual idiosyncrasies with this as with any other application. Flavine, unlike other antiseptics, is not reduced in power by contact with serum, and therefore the evaporation of fluid may result in a concentrated mass action upon the tissues. We have found that the pellicle in question is readily removed by the application of saline solution. The lethal concentration of flavine against cocci in the presence of serum being 1 in 200,000 (Browning), we find the addition of hypertonic saline solution a valuablo adjunct to the action of the antiseptic by producing free exudation; but allowing the fact of a microscopical layer of surface destruction, is it not a fact that all antiseptics are to some extent escharotic and produce in certain concentrations similar appearances? What we wish to kuow is-what is the exact clinical value of these observations: We are told that healing is delayed, that epithelial ingrowth is prevented, that organisms linger in the wound, and we find it extremely difficult to make these deductions from histological appearances square with our clinical experiences in thousands of cases. We are told gravely that " the employment of flavine and other dyes, as if they supplied royal roads to success, must be regarded as retro. grade steps," and we go round our wards and find patients with normal temperatures, with no signs of constitutional reaction, with healthy granulating wounds, over which evithelium is growing naturally, and we wonder wliy the experience of the authors of this article was so different in fifty cases from our own in as many hundreds.

DR. LUIGI LUCIANI, the distinguished professor of physiology in the University of Rome, lately retired under the rule as to limit of age. He was born at Ascoli-Piceno in 1842. He was professor of pathology at Parma, and of physiology at Siena and at Florence before his call to Rome in 1894. His treatise on physiology has been trans lated into several languages. A review of the last volume of the English translation was published on January 5 th. $\mathrm{He}$ is a Senator of the Kingdom of Italy, and as a Member of the Superior Council of Public Instruction he has done much during the past forty years for the improvement of medical education in his native country. 